Chirurg 2015 · 86:79

DOI 10.1007/s00104-014-2977-6

Online publiziert: 10. Januar 2015

(c) Springer-Verlag Berlin Heidelberg 2015

J. Reibetanz · C.-T. Germer

Klinik für Allgemein-, Viszeral-, Gefäß- und Kinderchirurgie, Universitätsklinikum Würzburg

\title{
Intensivierte adjuvante Chemotherapie mit FOLFOX beim multimodal behandelten Rektumkarzinom
}

\section{Originalpublikation}

Hong YS, Nam BH, Kim KP et al (2014) Oxaliplatin, fluorouracil, and leucovorin versus fluorouracil and leucovorin as adjuvant chemotherapy for locally advanced rectal cancer after preoperative chemoradiotherapy (ADORE): an open-label, multicentre, phase 2 , randomised controlled trial. Lancet Oncol 15(11):1245-1253

\section{Hintergrund}

Die neoadjuvante (Langzeit-)Radiochemotherapie gefolgt von der total mesorektalen Exzision (TME) ist die leitliniengerechte Standardtherapie des lokal fortgeschrittenen Rektumkarzinoms. Die Frage einer (grundsätzlichen) adjuvanten Chemotherapie für derart therapierte Patienten wird in der Literatur kontrovers diskutiert und durchgeführte Therapieprotokolle sind uneinheitlich. Während für das Kolonkarzinom gezeigt werden konnte, dass eine Oxaliplatin-basierte adjuvante Therapie (FOLFOX) gegenüber 5-Fluorouracil (5-FU) einen Überlebensvorteil im Stadium III bietet, gibt es keine belastbare Evidenz für eine derart intensivierte adjuvante Therapie für multimodal behandelte Patienten mit Rektumkarzinom.

\section{Fragestellung und Methode}

Die ADORE-Studie (ADjuvant Oxaliplatin in REctal cancer) ist eine randomisiert-kontrollierte, multizentrische Phase2-Studie zur Frage der therapeutischen Sicherheit und des onkologischen Nutzens von FOLFOX gegenüber 5-FU/Leucovorin in der adjuvanten Therapie von $\mathrm{Pa}$ tienten mit lokal fortgeschrittenem Rektumkarzinom nach neoadjuvanter Radio- chemotherapie. Hierzu wurden Patienten im postoperativen Tumorstadium II und III nach TME und bestätigter R0-Resektion 1:1-randomisiert in die Therapiearme FOLFOX (8 Zyklen) vs. 5-FU/Leucovorin (4 Zyklen). Primärer Endpunkt war das 3-Jahres-krankheitsfreie Überleben, sekundäre Endpunkte beinhalteten Therapiesicherheit/Nebenwirkungen, Lebensqualität und Gesamtüberleben.

\section{Ergebnisse}

Zwischen 2008 und 2012 wurden in 6 südkoreanischen Zentren 321 Patienten randomisiert, von denen die Daten von 295 Patienten ( $\mathrm{n}=146$ in der FOLFOX-Gruppe, $\mathrm{n}=149$ in der 5-FU-Gruppe) für die Auswertung zur Verfügung standen. Beide Kollektive waren vergleichbar hinsichtlich demographischer und klinischer Daten. 97\% der Patienten der FOLFOX-Gruppe und 95\% der 5-FU-Gruppe komplettierten alle geplanten Zyklen der adjuvanten Therapie. Nach einem medianen Followup von 38,2 Monaten $(26,2-50,6)$ lag das krankheitsfreie Überleben in der 5-FUGruppe bei 62,9\% (95\%-Konfidenzintervall[KI] 55,4-70,4) und in der FOLFOXGruppe bei 71,6\% (95\%-KI 64,6-78,6, Hazard Ratio [HR] 0,66, 95\%-KI 0,43-0,99, $\mathrm{p}=0,047)$. Auch das 3-Jahres-Gesamtüberleben war zugunsten der mit FOLFOX behandelten Patienten verlängert (5FU: $85,7 \%$ vs. FOLFOX: 95,0\%, HR 0,46, 95\%-KI 0,21-0,97, p=0,03). Das Gesamtnebenwirkungsprofil der Therapie (jeder Grad) war signifikant ungünstiger in der FOLFOX-Gruppe, jedoch zeigten sich keine signifikanten Unterschiede hinsichtlich schwerer (Grad 3/4) hämatologischer und nichthämatologischer Nebenwirkungen zwischen beiden Therapiearmen.

\section{Diskussion und Fazit des Reviewers}

Auch wenn es weiterhin eine leitliniengerechte Empfehlung ist, so konnte kürzlich durch die 10-Jahres-Ergebnisse der EORTC-22921-Studie gezeigt werden, dass eine alleinige 5-FU-basierte Adjuvanz wahrscheinlich nicht zu einer Prognoseverbesserung beim neoadjuvant vorbehandelten Rektumkarzinom führt [1]. Die aktuelle Arbeit gibt nun erste Hinweise auf einen onkologischen Benefit einer intensivierten adjuvanten Chemotherapie (FOLFOX) gegenüber 5-FU und bestätigt darüber hinaus, dass die Hinzunahme von Oxaliplatin in das postoperative Behandlungsprotokoll - trotz erhöhter Gesamtrate an Nebenwirkungen - die Therapiecompliance nicht negativ beeinflusst. Die wichtige Frage, ob überhaupt (und wenn ja, in welchem pathohistologischen Tumorstadium) eine adjuvante Therapie sinnvoll ist, kann diese Studie jedoch nicht beantworten.

\section{Korrespondenzadresse}

\section{Prof. Dr. C.-T. Germer}

Klinik für Allgemein-, Viszeral-, Gefäß- und Kinderchirurgie, Universitätsklinikum Würzburg, Oberdürrbacher Str. 6, 97080 Würzburg germer_c@ukw.de

Interessenkonflikt. J. Reibetanz und C.-T. Germer geben an, dass kein Interessenkonflikt besteht.

\section{Literatur}

1. Bosset JF, Calais G, Mineur L et al (2014) Fluorouracil-based adjuvant chemotherapy after preoperative chemoradiotherapy in rectal cancer: longterm results of the EORTC 22921 randomised study. Lancet Oncol 15:184-190 